\title{
Multifunctions of Souslin type: Corrigendum
}

\section{S.J. Leese}

In [1], Theorem 6, a sufficient condition is given for a multifunction to be "of Souslin type". However, the proof contains an error; we are required to prove that the multifunction $\Omega: S \rightarrow P \times N^{N}$ defined by

$$
G(\Omega)=\bigcup_{\sigma} \prod_{n=1}^{\infty}\left[A_{\sigma \mid n} \times\left(B_{\sigma \mid n}^{*} \times C_{\sigma \mid n}\right)\right]
$$

has values which are closed subsets of $P \times N^{N}$ (the notation is explained in [1]). The "proof" of this fact given in [1] is manifestly incorrect as it appears to assume that $N^{N}$ has the discrete topology. Instead we use the fact that, for a fixed sequence $\sigma$ of positive integers, the sets $\left\{C_{\sigma \mid n}: n=1,2, \ldots\right\}$ form a base of neighbourhoods of $\sigma$.

Suppose then that $(x, \tau) \vDash \Omega(t)$. Then

$$
(t, x, \tau) \notin \bigcap_{n=1}^{\infty}\left(A_{\tau \mid n} \times B_{\tau \mid n}^{*} \times C_{\tau \mid n}\right)=\bigcap_{n=1}^{\infty}\left(A_{\tau \mid n} \times B_{\tau \mid n}^{*}\right) \times\{\tau\},
$$

and so there is an integer $n$ such that $(t, x)$ does not belong to ${ }^{A}{ }_{\tau \mid n} \times B_{\tau \mid n}^{*}$. There are two cases to consider. Firstly, if $t$ does not belong to $A_{\tau \mid n}$, the reader may verify that the neighbourhood $P \times C_{\tau \mid n}$ of $(x, \tau)$ does not meet $\Omega(t)$. If $t$ does belong to $A_{\tau \mid n}$, there is a neighbourhood $U \times C_{\tau \mid n}$ of $(x, \tau)$ which does not meet $\Omega(t)$. Therefore the set $\Omega(t)$ is closed.

Received 20 May 1975. 


\section{Reference}

[1] S.J. Leese, "Multifunctions of Souslin type", BulZ. Austral. Math. Soc. $11(1974), 395411$.

Department of Mathematics,

Loughborough University of Technology,

Loughborough,

Leicestershire,

England. 